\title{
Observer Design for Simultaneous State and Fault Estimation for a Class of Discrete-time Descriptor Linear Models
}

\author{
Kaoutar Ouarid ${ }^{1,2, *}$, Abdellatif El Assoudi ${ }^{1,2, * *}$, Jalal Soulami ${ }^{1,2, * * *}$, and El Hassane El Yaagoubi ${ }^{1,2, * * * *}$ \\ ${ }^{1}$ Laboratory of High Energy Physics and Condensed Matter, Faculty of Science Hassan II University of Casablanca. \\ B.P 5366, Maarif, Casablanca, Morocco \\ ${ }^{2}$ ECPI, Department of Electrical Engineering, ENSEM Hassan II University of Casablanca. \\ B.P 8118, Oasis, Casablanca Morocco
}

\begin{abstract}
This paper investigates the problem of observer design for simultaneous states and faults estimation for a class of discrete-time descriptor linear models in presence of actuator and sensor faults. The idea of the present result is based on the second equivalent form of implicit model [1] which permits to separate the differential and algebraic equations in the considered singular model, and the use of an explicit augmented model structure. At that stage, an observer is built to estimate simultaneously the unknown states, the actuator faults, and the sensor faults. Next, the explicit structure of the augmented model is established. Then, an observer is built to estimate simultaneously the unknown states, the actuator faults, and the sensor faults. By using the Lyapunov approach, the convergence of the state estimation error of the augmented system is analyzed, and the observer's gain matrix is achieved by solving only one linear matrix inequality (LMI). At long last, an illustrative model is given to show the performance and capability of the proposed strategy.
\end{abstract}

\section{Introduction}

With the emergence of the industry of the future, many industrial processes have become equipped with very sophisticated sensors in order to collect all the information relating to the discovery of any unexpected change that may occur in the process. As a result, it has become so necessary to guarantee an effective control, and corrective actions in order to maintain the best performance of the process in terms of efficiency, reliability, availability, safety and security [2-4].

As any technical system governed by both dynamic and static equations, it needs to be modeled in its study. Generally, the implicit model, otherwise called the descriptor or singular model [1, 5, 6], has become the most common in the description of the behavior of the process.

Thus, to maintain production continuity, high throughput, and good performance of the industrial process, it has become essential to use detection and diagnostic tools to identify faults in the process, and in any other equipment which is capable of contributing to the degradation of the performance of the entire process. Due to its many advantages, the fault detection and diagnosis (FDD) have attracted the attention of academia as well as the industry in various fields, for instance, [7-12].

\footnotetext{
*e-mail: kaoutar.ouarid@gmail.com

**e-mail: a.elassoudi@ensem.ac.ma

***e-mail: jalal.soulami@gmail.com

****e-mail: h.elyaagoubi@ensem.ac.ma
}

In the literature, several FDD methods have been used which can be classified into knowledge-based methods, data-based methods and finally model-based methods that are well known [9, 13-18]. Between the techniques used in the latter ones, we find these based on observers [19-29].

In this perspective, the aim of this work is to study the problem of observer design in order to propose actuator, and sensor faults estimation method for a class of discrete-time descriptor linear models.This problem has been widely tackled and many research works are devoted to this class of systems. They relate to explicit and singular linear models in both continuous and discrete-time cases [30-40]. The resolution of the design and stability problems of the observer lies in the writing of the convergence conditions in the form of LMIs solved by a dedicated software [41].

In this paper, a novel approach for designing an observer for a class of discrete-time descriptor linear models subject to actuator and sensor faults is proposed. To achieve this, we propose a new method for simultaneous state, and fault estimation that is based on the separation between differential and algebraic equations in the considered descriptor model as given in [1]. More precisely, based on an explicit augmented model structure, observer design for a class of discrete-time descriptor linear models allows the simultaneous estimation of the unknown states, the actuator faults and the sensor faults is proposed. The exponential stability of the state estimation error is studied 
is given in term of LMI. Besides, the proposed result is synthesized by only an explicit structure.

The rest of this article is arranged as follows. In the second section, the class of discrete-time linear implicit models with actuator and sensor faults is presented. In the third section, the main result about observer design permits to estimate simultaneously unmeasurable states, and unknown faults of actuator and sensor is stated. In the fourth section, we illustrate the performance of the developed result in simulation through an implicit model of a heat exchanger pilot process. Finally, the conclusion is given in the fifth section.

Through this paper, the notations are similar to these used in [27] with the purpose of simplifying its structure.

\section{Model description}

The class of descriptor linear models considered is presented as follows:

$$
\left\{\begin{array}{cl}
\mathbf{E} Z_{k+1} & =A Z_{k}+B u_{k}+F_{a} f_{a k} \\
y_{k} & =C Z_{k}+D u_{k}+D_{a} f_{a k}+F_{s} f_{s k}
\end{array}\right.
$$

where: The state vector is presented such as $Z_{k}=$ $\left[Z_{k}^{1 T} Z_{k}^{2 T}\right]^{T} \in \mathbb{R}^{n}$ with $Z_{k}^{1} \in \mathbb{R}^{r}$ is the vector of differential variables, $Z_{k}^{2} \in \mathbb{R}^{n-r}$ is the vector of algebraic variables, $u_{k} \in \mathbb{R}^{m}$ is the control input, $y_{k} \in \mathbb{R}^{p}$ is the measured output vector. $f_{a k} \in \mathbb{R}^{n_{a}}$ and $f_{s k} \in \mathbb{R}^{n_{s}}$ are the actuator fault and sensor fault, respectively.

$A \in \mathbb{R}^{n \times n}, B \in \mathbb{R}^{n \times m}, C \in \mathbb{R}^{p \times n}, D \in \mathbb{R}^{p \times m}, D_{a} \in \mathbb{R}^{p \times n_{a}}$, $\mathbf{E} \in \mathbb{R}^{n \times n}, F_{a} \in \mathbb{R}^{n \times n_{a}}$ and $F_{s} \in \mathbb{R}^{p \times n_{s}}$, are real known constant matrices with:

$$
\begin{gathered}
A=\left(\begin{array}{ll}
A_{11} & A_{12} \\
A_{21} & A_{22}
\end{array}\right) ; \quad B=\left(\begin{array}{l}
B_{1} \\
B_{2}
\end{array}\right) \\
F_{a}=\left(\begin{array}{c}
F_{a}^{1} \\
F_{a}^{2}
\end{array}\right) ; \quad C=\left(\begin{array}{ll}
C_{1} & C_{2}
\end{array}\right)
\end{gathered}
$$

where: The constant matrix $A_{22}$ is supposed invertible. The matrix $\mathbf{E}$ is assumed to be of the form:

$$
\mathbf{E}=\left(\begin{array}{ll}
I & 0 \\
0 & 0
\end{array}\right)
$$

and satisfies $\operatorname{rank}(\mathbf{E})=r<n$. If it is not the case, without loss of generality, we can always find $\Lambda$ and $\Omega$ such that $\mathbf{E}$ can be transformed into the form (4). That is:

$$
\Lambda \mathbf{E} \Omega=\left(\begin{array}{ll}
I & 0 \\
0 & 0
\end{array}\right)
$$

Before giving the main result, let us make the following assumption [1]:

\section{Assumption 1 : Suppose that:}

- $(\mathbf{E}, A)$ is regular, i.e. $\operatorname{det}(s \mathbf{E}-A) \neq 0 \forall s \in \mathbb{C}$

- System (I) is impulse observable and detectable. scriptor linear model (1), the approach is based on the separation between differential and algebraic equations in model (1).

So, using (2), (3) and (4), model (1) can be rewritten as follows:

$$
\left\{\begin{array}{c}
Z_{k+1}^{1}=A_{11} Z_{k}^{1}+A_{12} Z_{k}^{2}+B_{1} u_{k}+F_{a}^{1} f_{a k} \\
0=A_{21} Z_{k}^{1}+A_{22} Z_{k}^{2}+B_{2} u_{k}+F_{a}^{2} f_{a k} \\
y_{k}=C_{1} Z_{k}^{1}+C_{2} Z_{k}^{2}+D u_{k}+D_{a} f_{a k}+F_{s} f_{s k}
\end{array}\right.
$$

The form (6) for system (1) is also known as the second equivalent form [1].

From (6) and using the fact that $\left(A_{22}\right)^{-1}$ exist, the algebraic equations can be solved directly for algebraic variables, to obtain:

$$
Z_{k}^{2}=J Z_{k}^{1}+K u_{k}+L_{a} f_{a k}
$$

where:

$$
\left\{\begin{aligned}
J & =-\left(A_{22}\right)^{-1} A_{21} \\
K & =-\left(A_{22}\right)^{-1} B_{2} \\
L_{a} & =-\left(A_{22}\right)^{-1} F_{a}^{2}
\end{aligned}\right.
$$

Substitution of the resulting expression for $Z_{k}^{2}$ (equation (7) in equation (6) yields the following model:

$$
\left\{\begin{array}{cl}
Z_{k+1}^{1} & =M Z_{k}^{1}+N u_{k}+P_{a} f_{a k} \\
Z_{k}^{2} & =J Z_{k}^{1}+K u_{k}+L_{a} f_{a k} \\
y_{k} & =R Z_{k}^{1}+S u_{k}+T_{a} f_{a k}+F_{s} f_{s k}
\end{array}\right.
$$

where:

$$
\left\{\begin{array}{c}
M=A_{11}+A_{12} J \\
N=B_{1}+A_{12} K \\
P_{a}=F_{a}^{1}+A_{12} L_{a} \\
R=C_{1}+C_{2} J \\
S=D+C_{2} K \\
T_{a}=D_{a}+C_{2} L_{a}
\end{array}\right.
$$

Which is equivalent to the following state representation:

$$
\left\{\begin{array}{cc}
Z_{k+1}^{1} & =M Z_{k}^{1}+N u_{k}+P \epsilon_{k} \\
Z_{k}^{2} & =J Z_{k}^{1}+K u_{k}+L \epsilon_{k} \\
y_{k} & =R Z_{k}^{1}+S u_{k}+T \epsilon_{k}
\end{array}\right.
$$

where:

$$
\left\{\begin{aligned}
\epsilon_{k} & =\left(\begin{array}{c}
f_{a k} \\
f_{s k}
\end{array}\right) \\
P & =\left(\begin{array}{ll}
P_{a} & 0
\end{array}\right) \\
L & =\left(\begin{array}{ll}
L_{a} & 0
\end{array}\right) \\
T & =\left(\begin{array}{ll}
T_{a} & F_{s}
\end{array}\right)
\end{aligned}\right.
$$

Assumption 2 : Suppose that the fault $\epsilon_{k}$ is considered as a constant unknown fault signal per time interval i.e.:

$$
\epsilon_{k+1} \quad=\quad \epsilon_{k} \quad k \in\left[\begin{array}{ll}
T_{1} & T_{2}
\end{array}\right] \quad ; \forall T_{1}, T_{2} \in \mathbb{R}^{+}
$$


the system (11) under the equivalent augmented state representation given by:

$$
\left\{\begin{array}{c}
\chi_{k+1}^{1}=\tilde{M} \chi_{k}^{1}+\tilde{N} u_{k} \\
\chi_{k}^{2}=\tilde{J} \chi_{k}^{1}+K u_{k} \\
y_{k}=\tilde{R} \chi_{k}^{1}+S u_{k}
\end{array}\right.
$$

where:

$$
\left\{\begin{aligned}
\chi_{k}^{1} & =\left(\begin{array}{c}
Z_{k}^{1} \\
\epsilon_{k}
\end{array}\right) \\
\chi_{k}^{2} & =Z_{k}^{2} \\
\tilde{M} & =\left(\begin{array}{cc}
M & P \\
0 & I
\end{array}\right) \\
\tilde{N} & =\left(\begin{array}{c}
N \\
0
\end{array}\right) \\
\tilde{J} & =\left(\begin{array}{ll}
J & L \\
R & T
\end{array}\right)
\end{aligned}\right.
$$

\section{Simultaneous State and Fault Estimation}

Based on the transformation of the implicit model (1) into the equivalent form (14), the proposed observer permitting to estimate simultaneously unmeasurable state and unknown faults and their derivatives takes the following form:

$$
\left\{\begin{aligned}
\hat{\chi}_{k+1}^{1} & =\tilde{M} \hat{\chi}_{k}^{1}+\tilde{N} u_{k}+G\left(y_{k}-\hat{y}_{k}\right) \\
\hat{\chi}_{k}^{2} & =\tilde{J} \hat{\chi}_{k}^{1}+K u_{k} \\
\hat{y}_{k} & =\tilde{R} \hat{\chi}_{k}^{1}+S u_{k}
\end{aligned}\right.
$$

where: $\left(\hat{\chi}_{k}^{1}, \hat{\chi}_{k}^{2}\right)$ and $\hat{y}_{k}$ denote the estimated augmented state vector and the output vector respectively. $G$ is the observer gain which is determined such that $\left(\hat{\chi}_{k}^{1}, \hat{\chi}_{k}^{2}\right)$ asymptotically converges to $\left(\chi_{k}^{1}, \chi_{k}^{2}\right)$.

In order to establish the condition for the asymptotic convergence of the observer (16), we define the state estimation error:

$$
e_{k}=\left(\begin{array}{c}
e_{k}^{1} \\
e_{k}^{2}
\end{array}\right)=\left(\begin{array}{c}
\chi_{k}^{1}-\hat{\chi}_{k}^{1} \\
\chi_{k}^{2}-\hat{\chi}_{k}^{2}
\end{array}\right)
$$

It follows, from (14) and (16), the dynamics of state estimation error $e_{k}$ is given by the differential and algebraic equations:

$$
\left\{\begin{array}{cc}
e_{k+1}^{1} & =\Psi e_{k}^{1} \\
e_{k}^{2} & =\tilde{J} e_{k}^{1}
\end{array}\right.
$$

where

$$
\Psi=\tilde{M}-G \tilde{R}
$$

Note that, to prove the convergence of the estimation error $e_{k}$ toward zero, it suffices to prove from (18), that $e_{k}^{1}$ converges toward zero.

Theorem 1 There exists an observer (16) for the descriptor linear model (1) if for a scalar $\alpha>0$, there exists matrices $P>0$ and $W$ such that the following LMI holds:

$$
\left(\begin{array}{cc}
-\alpha^{2} P & * \\
P \tilde{M}-W \tilde{R} & -P
\end{array}\right)<0
$$

The gain $G$ of the observer is derived from:

$$
G=P^{-1} W
$$

quadratic Lyapunov function as follows:

$$
V_{k}=e_{k}^{1 T} P e_{k}^{1}, \quad P>0
$$

Its time derivative is given by:

$$
\begin{aligned}
V_{k+1}-V_{k} & =e_{k}^{1 T}\left(\Psi^{T} P \Psi-P\right) e_{k}^{1} \\
& <\left(\alpha^{2}-1\right) V_{k}
\end{aligned}
$$

Thus, estimation error convergence is exponentially ensured if the following condition is guaranteed:

$$
e_{k}^{1 T}\left(\Psi^{T} P \Psi-\alpha^{2} P\right) e_{k}^{1}<0 ; \alpha>0
$$

Which is equivalent to the following condition:

$$
\Psi^{T} P \Psi-\alpha^{2} P<0
$$

Replacing $\Psi$ from (19) into (25), we can establish the LMI condition (20) of Theorem 1 by using the following change of variable:

$$
W=P G
$$

Thus, from the Lyapunov stability theory, if the LMI condition (20) is satisfied, the error dynamic equation (18) is exponentially asymptotically stable. This completes the proof of Theorem 1

\section{Application to a heat exchanger pilot process}

In this section, the theory of the proposed observer (16) is applied to a heat exchanger pilot process with the aim of simultaneously estimating the unknown states and faults on-line. The following discrete-time model that we consider here is obtained by Euler discretisation of step size $\mathrm{T}=0.012 \mathrm{~s}$ of the model given in [27]. It takes the form:

$$
\left\{\begin{array}{c}
\mathbf{E} Z_{k+1}=A Z_{k}+B u_{k}+F_{a} f_{a k} \\
y_{k}=C Z_{k}+D u_{k}+F_{s} f_{s k}
\end{array}\right.
$$

where: $Z_{k}=\left[Z_{1 k}, \ldots, Z_{8 k}\right]^{T} \in \mathbb{R}^{8}$ is the state vector, $u_{k} \in \mathbb{R}^{2}, y_{k} \in \mathbb{R}^{2}, f_{a k} \in \mathbb{R}$ and $f_{s k} \in \mathbb{R}$ are the control vector, the vector of output measurements, the actuator fault, and the sensor fault, respectively. The $\operatorname{rank}(\mathbf{E})=6$ and the numerical values of the matrices are given by:

$$
\begin{aligned}
A_{11} & =\left(\begin{array}{cccccc}
0.9829 & 66.3104 & 0 & 0.0034 & 0 & 0 \\
0 & 1.0000 & 0.0120 & 0 & 0 & 0 \\
0 & 0 & 1.0000 & 0 & 0 & 0 \\
0.0011 & 0 & 0 & 0.9943 & -11.0517 & 0 \\
0 & 0 & 0 & 0 & 1.0000 & 0.0120 \\
0 & 0 & 0 & 0 & 0 & 1.0000
\end{array}\right) \\
A_{21} & =\left(\begin{array}{cccccc}
0 & -0.4737 & -0.1056 & 0 & 0 & 0 \\
0 & 0 & 0 & 0 & -0.4737 & -0.1056
\end{array}\right)
\end{aligned}
$$$$
A_{12}=\left(\begin{array}{cc}
0 & 0 \\
0 & 0 \\
0.0120 & 0 \\
0 & 0 \\
0 & 0 \\
0 & 0.0120
\end{array}\right) ; A_{22}=\left(\begin{array}{cc}
-0.0120 & 0 \\
0 & -0.0120
\end{array}\right)
$$ 


$$
\begin{aligned}
& B_{1}=\left(\begin{array}{ll}
0 & 0 \\
0 & 0 \\
0 & 0 \\
0 & 0 \\
0 & 0 \\
0 & 0
\end{array}\right) ; \quad B_{2}=\left(\begin{array}{cc}
0.4406 & 0 \\
0 & 0.4406
\end{array}\right) \\
& F_{a}^{1}=\left(\begin{array}{l}
0 \\
0 \\
0 \\
0 \\
0 \\
0
\end{array}\right) ; \quad F_{a}^{2}=\left(\begin{array}{c}
0.4406 \\
0
\end{array}\right) \\
& F_{s}=\left(\begin{array}{c}
1 \\
0
\end{array}\right) ; D=\left(\begin{array}{cc}
0 & 0 \\
0 & 0
\end{array}\right) \\
& C_{1}=\left(\begin{array}{cccccc}
1 & 0 & 0 & 0 & 0 & 0 \\
0 & 0 & 0 & 1 & 0 & 0
\end{array}\right) ; \quad C_{2}=\left(\begin{array}{cc}
0 & 0 \\
0 & 0
\end{array}\right)
\end{aligned}
$$

In the following simulation results, under assumption 2 , faults $f_{a k}$ and $f_{s k}$ are defined as given in figure 5 and can be applied in the same time.

Therefore, to apply the proposed observer design (16) for the implicit model (27), as stated in Theorem 1, it suffices to rewrite the model (27) into its equivalent form (14) as mentioned in Section 2.

Thus, the resolution of the LMI defined in Theorem 1 with $\alpha=0.98$ lead the following numerical value:

$$
G=\left(\begin{array}{cc}
1.9177 & 804.3812 \\
0.0161 & 0.2076 \\
0.0392 & -0.0023 \\
0.0011 & 2.5371 \\
-0.0000 & -0.1051 \\
0.0000 & -0.3066 \\
0.0149 & 0.2235 \\
0.1003 & -804.3834
\end{array}\right)
$$

Figures 1 to 5 show the results of the simulation when actuator and sensor faults, shown in figure 1, occur. In fact, the estimated states of the the heat exchanger pilot process 27) are represented by the Figures 2 to 5 .

It is easy to see that even when a fault affects the system, the proposed observer, designed by using the numerical value of the gain (28), gives a good performance which allow the estimated signals, denoted by the dashed lines, to converge to the real signals.
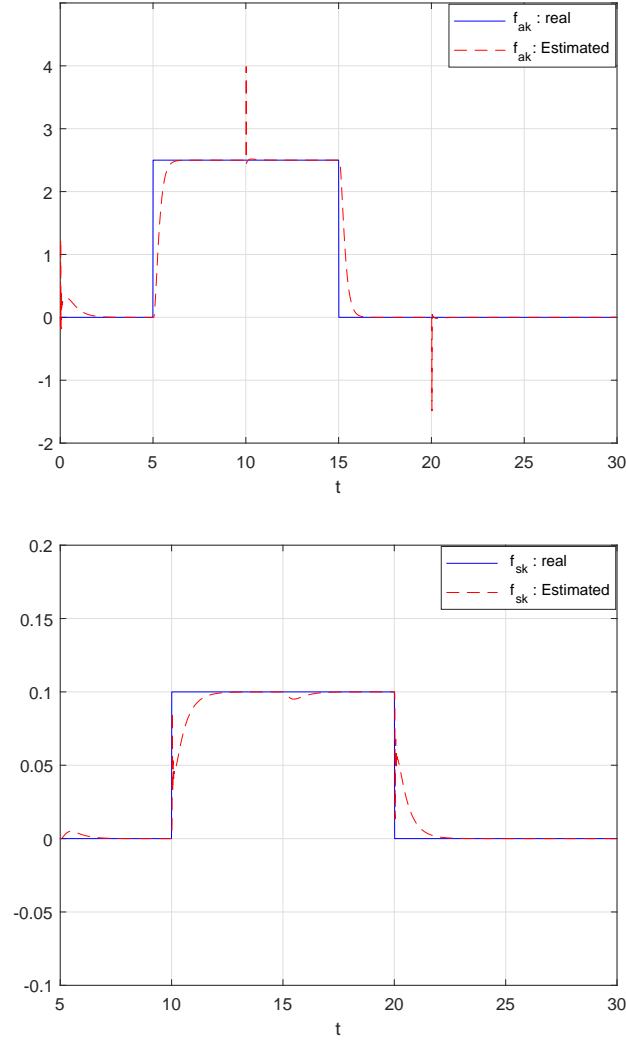

Figure 1. $f_{a k}$ and $f_{s k}$ with their estimates
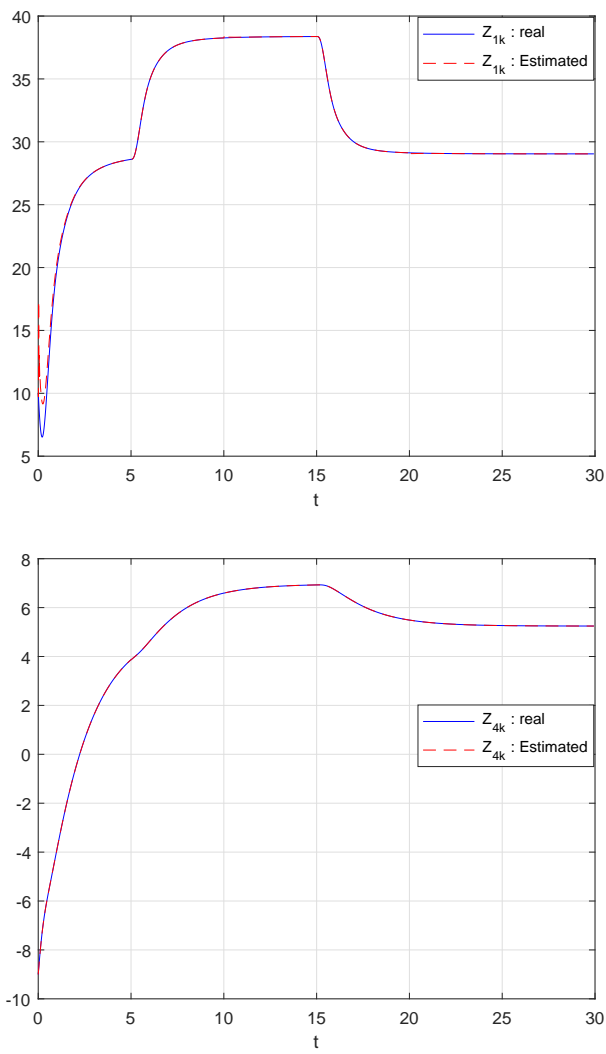

Figure 2. $Z_{1 k}$ and $Z_{4 k}$ with their estimates 

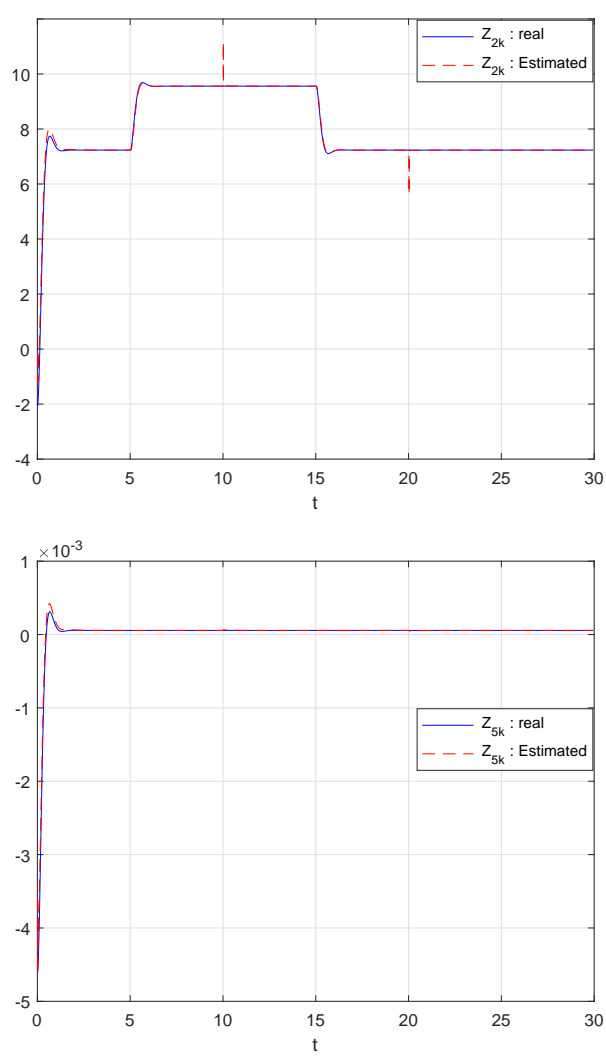

Figure 3. $Z_{2 k}$ and $Z_{5 k}$ with their estimates
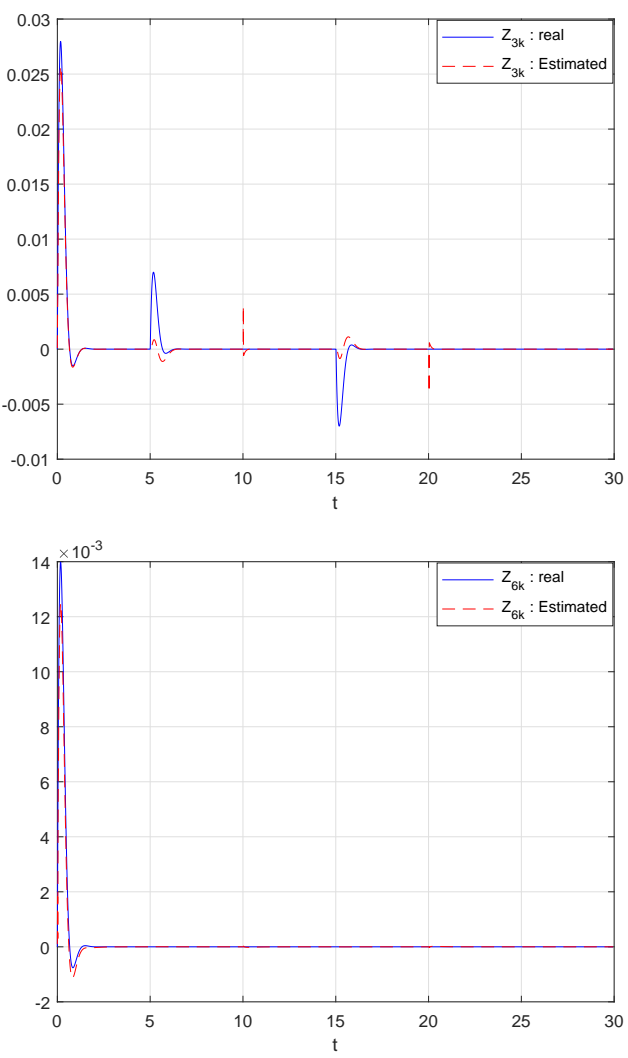

Figure 4. $Z_{3 k}$ and $Z_{6 k}$ with their estimates
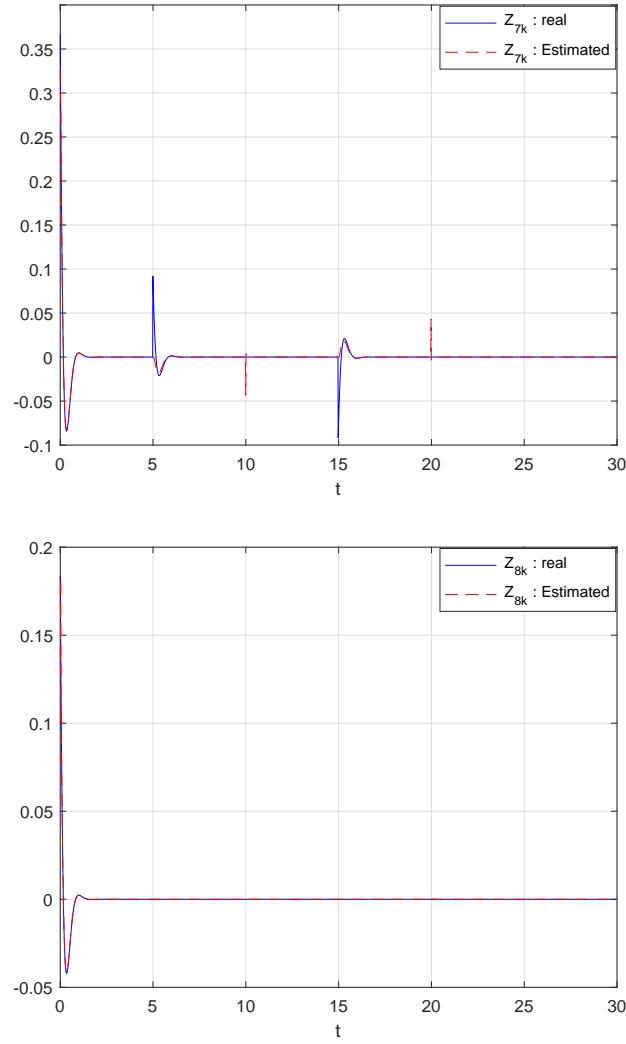

Figure 5. $Z_{7 k}$ and $Z_{8 k}$ with their estimates

\section{Conclusion}

A new observer design approach for simultaneous state and fault estimation for a class of discrete-time descriptor linear models is proposed in this paper. The main idea of the present work is based on the decomposition approach in which the observer parameters are determined by using the Lyapunov theory and solving a system of LMI. It presents the extension of the work carried out in continuous case [27] to the discrete case. The good performance of the proposed observer is shown in simulation through a heat exchanger process as an illustrative application.

\section{References}

[1] L. Dai, Singular Control Systems, Berlin, Germany: Springer (1989). https://doi.org/10.1007/BFb0002476

[2] Y.-J. Park, S.-K. S. Fan, C.-Y. Hsu, A Review on Fault Detection and Process Diagnostics in Industrial Processes. Processes, 8(9), 1123 (2020). https://doi.org/10.3390/pr8091123

[3] L. Ming, J. Zhao, Review on chemical process fault detection and diagnosis, 6th International Symposium on Advanced Control of Industrial Processes (AdCONIP) (2017). https://do1.org/10.1109/adconip.2017.7983824 
Based Condition Monitoring: Actuators, Drives, Machinery, Plants, Sensors, and Fault-tolerant Systems, Springer-Verlag Berlin Heidelberg (2011). https://doi.org/10.1007/978-3-642-12767-0_2

[5] A. Kumar, P. Daoutidis, Control of nonlinear differential algebraic equation systems, Chapman and Hall CRC (1998).

[6] G.-R. Duan, Analysis and Design of Descriptor Linear Systems, New York, NY: Springer (2010).

[7] R. Isermann, Fault-Diagnosis Systems: an Introduction from Fault Detection to Fault Tolerance, 1, Springer Science and Business Media (2006). https://doi.org/10.1007/3-540-30368-5

[8] Z. Lendek, T. M. Guerra, R. Babuška and B. De Schutter, Stability Analysis and Nonlinear Observer Design Using Takagi-Sugeno Fuzzy Models, Studies in Fuzziness and Soft Computing, Springer-Verlag Berlin Heidelberg (2011). https://doi.org/10.1007/978-3-642-16776-8

[9] D. Miljkovic, Fault detection methods: a literature survey, in: Proceedings of the 34th International Convention MIPRO, pp. 750-755 (2011).

[10] M. Witczak, Fault Diagnosis and Fault-Tolerant Control Strategies for Non-Linear Systems, Analytical and Soft Computing Approaches, Springer International Publishing Switzerland (2014). https://doi.org/10.1007/978-3-319-03014-2

[11] M. Blanke, M. Kinnaert, J. Lunze, M. Staroswiecki, Diagnosis and Fault-Tolerant Control. Springer-Verlag Berlin Heidelberg (2016). https://doi.org/10.1007/978-3-662-47943-8

[12] L. Li, Fault Detection and Fault-Tolerant Control for Nonlinear Systems. Springer Fachmedien Wiesbaden (2016). https://doi.org/10.1007/978-3-658-13020-6

[13] V. Venkatasubramanian, R. Rengaswamy, K. Yin, S.N. Kavuri, A review of process fault detection and diagnosis part i: quantitative model-based methods, Comput. Chem. Eng. 27 (3) 293-311 (2003). https://doi.org/10.1016/S0098-1354(02)00160-6

[14] V. Venkatasubramanian, R. Rengaswamy, S.N. Kavuri, A review of process fault detection and diagnosis part ii : qualitative models and search strategies, Comput. Chem. Eng. 27 (3) 313-326 (2003). https://doi.org/10.1016/S0098-1354(02)00161-8

[15] V. Venkatasubramanian, R. Rengaswamy, S.N. Kavuri, K. Yin, A review of process fault detection and diagnosis part iii: process history based methods, Comput. Chem. Eng. 27 (3) 327-346 (2003). https://doi.org/10.1016/s0098-1354(02)00162-x

[16] Z. Gao, C. Cecati, S.X. Ding, A survey of fault diagnosis and fault-tolerant techniques-part I: fault diagnosis with model-based and signal-based approaches, IEEE Trans. Ind. Electron. 62 (6) 3757-3767 (2015). https://doi.org/10.1109/TIE.2015.2417501

[17] I. Hwang, S. Kim, Y. Kim, C.E. Seah, A Survey of Fault Detection, Isolation, and Reconfiguration Methods, IEEE Transactions on Con- https://doi.org/10.1109/tcst.2009.2026285

[18] Z. Gao, C. Cecati, S.X. Ding, A survey of fault diagnosis and fault-tolerant techniques-part II: fault diagnosis with knowledge-based and hybrid/active approaches, IEEE Trans. Ind. Electron. 62 (6) 3768-3774 (2015). https://doi.org/10.1109/TIE.2015.2419013

[19] J. Chen, R.J. Patton, Diagnosis of Non-Linear Dynamic Systems, The International Series on Asian Studies in Computer and Information Science, vol 3. Springer, Boston, MA (1999). https://doi.org/10.1007/978-1-4615-5149-2_9

[20] J. Qiu, M. Ren, Y. Niu, Y. Zhao, Y. Guo, Fault Estimation for Nonlinear Dynamic Systems, Circuits, Systems, and Signal Processing, 31(2), 555-564 (2011). https://doi.org/10.1007/s00034-011-9348-z

[21] H. Hamdi, M. Rodrigues, C. Mechmeche, N.B. Braiek, Robust fault detection and estimation for descriptor systems based on multi-models concept, International Journal of Control, Automation and Systems, 10(6), 1260-1266 (2012). https://doi.org/10.1007/s12555-012-0622-z

[22] Y. Yang, S.X. Ding, L. Li, On observerbased fault detection for nonlinear systems, Systems and Control Letters, 82, 18-25 (2015). https://doi.org/10.1016/j.sysconle.2015.05.004

[23] T. Youssef, M. Chadli, H.R. Karimi, R. Wang, Actuator and sensor faults estimation based on proportional integral observer for TS fuzzy model, J. Frankl. Inst. 354 (6) 2524-2542 (2017). https://doi.org/10.1016/j.jfranklin.2016.09.020

[24] K. Ouarid, A .El Assoudi, J. Soulami, E. El Yaagoubi, Fault Estimation Based on the Observer Design for Discrete-time Takagi-Sugeno Descriptor Models, 2019 Third International Conference on Intelligent Computing in Data Sciences (ICDS) (2019). https://doi.org/10.1109/icds47004.2019.8942301

[25] H. Han, Y. Yang, L. Li, S.X. Ding, Observerbased fault detection for uncertain nonlinear systems, J. Frankl. Inst. 355 (3) 1278-1295 (2018). https://doi.org/10.1016/j.jfranklin.2017.12.021

[26] M.F. Pico, E.J. Adam, Fault diagnosis and tolerant control using observer banks applied to continuous stirred tank reactor, Adv. Sci. Technol. Eng. Syst. J. 2 (3) 171-181 (2017). https://doi.org/10.25046/aj020322

[27] K. Ouarid, A. El Assoudi, J. Soulami, E. El Yaagoubi, Observer Design for Simultaneous State and Fault Estimation for a Class of Continuoustime Implicit Linear Models, IEEE The International Conference of Computer Science and Renewable Energies, 978-1-7281-0826-1/19 (2019). https://doi.org/10.1109/ICCSRE.2019.8807633

[28] M. A. Eissa, A. Sali, M. k. Hassan, A.M. Bassiuny, R. R. Darwish, Observer-Based Fault Detection With Fuzzy Variable Gains and Its Application to Industrial Servo System,IEEE Access (2020). https://doi.org/10.1109/ACCESS.2020.3010125 
Yaagoubi, Design of Fuzzy Observer for a Class of Takagi-Sugeno Descriptor Models to Simultaneously Estimate States and Faults, Journal of Advanced Research in Dynamical and Control Systems (2020). https://doi.org/10.5373/JARDCS/V12SP5/20201754

[30] M. Darouach, M. Boutayeb, Design of observers for descriptor systems, IEEE Transactions on Automatic Control, vol. 40, pp. 1323-1327 (1995). https://doi.org/10.1109/9.400467

[31] D. Koenig, Unknown Input Proportional MultipleIntegral Observer Design for Linear Descriptor Systems: Application to State and Fault Estimation, IEEE Transactions on Automatic Control, Vol. 50, No. 2 (2005). https://doi.org/10.1109/TAC.2004.841889

[32] Z. Gao, S. X. Ding, Y. Ma, Robust fault estimation approach and its application in vehicle lateral dynamic systems, Optimal Control Applications and Methods, vol. 28, no. 3, pp. 143-156 (2007). https://doi.org/10.1002/oca.786

[33] Z. Wang, Y. Shen, X. Zhang, Q. Wang. Observer design for discrete-time descriptor systems : An LMI approach, Systems and Control Letters, 61(6) :683-687 (2012). https://doi.org/10.1016/j.sysconle.2012.03.006

[34] M. Darouach, On the functional observers for linear descriptor systems, Syst. Control Lett., vol. 61, no. 3, pp. 427-434 (2012). https://doi.org/10.1016/j.sysconle.2012.01.006

[35] Q. Jia, H. Li, Y. Zhang, X. Chen Robust Observer-based Sensor Fault Reconstruction for Discrete-Time Systems via a Descriptor System Approach, International Journal of Control, Automation and Systems 13(2):1-10 (2015).
[36] G.-L. Osorio-Gordillo, M. Darouach, C.-M. Astorga-Zaragoza, L. Boutat-Baddas, Fault diagnosis for discrete-time descriptor linear systems, International Federation of Automatic Control, vol. 48, no. 21, 1238-1243 (2015). https://doi.org/10.1016/j.ifacol.2015.09.695

[37] Z. Wang, M. Rodrigues, D. Theilliol, Y. Shen, Fault estimation Filter design for discrete-time descriptor systems, IET Control Theory and Applications, Institution of Engineering and Technology, 9(10), pp.15871594 (2015). https://doi.org/10.1049/iet-cta.2014.0641

[38] Z. Gao, Fault estimation and fault tolerant control for discrete-time dynamic systems, IEEE Transactions on Industrial Electronics, 62(6), 3874-3884 (2015). https://doi.org/10.1109/TIE.2015.2392720

[39] I. Haj Brahim, M. Chaabane, G. Hashim and D. Mehdi, Sensor Fault and State Estimation for Continuous-Time Descriptor Linear Systems: an LMI Approach. Proceedings of the 2016 5th International Conference on Systems and Control, Cadi Ayyad University, Marrakech, Morocco, May 25-27 (2016). https://doi.org/10.1109/ICoSC.2016.7507057

[40] J. Zhang, F. Zhu, J. Li, X. Li, Discrete-time linear descriptor system unknown input observer design: an auxiliary output-based approach, International Journal of Control, Automation and Systems, 15(6), 2599-2607 (2017). https://doi.org/10.1007/s12555-016-0611-8

[41] S. Boyd, L. E. Ghaoui, E. Feron, V. Balakrishnan, Linear Matrix Inequalities in Systems and Control Theory, Philadelphia, PA: SIAM (1994). https://doi.org/10.1137/1.9781611970777 